\title{
Evaluation of Neck Mass by Multidetector Computed Tomography and Histopathological Comparison.
}

\author{
Siddiqua UI ${ }^{1}$, Halder B ${ }^{2}$, Hossain $\mathrm{MR}^{2}$, Roy $\mathrm{SK}^{2}$, Al-Amin $\mathrm{A}^{2}$, Sultana $\mathrm{S}^{3}$ \\ ${ }^{1}$ Department of Radiology and Imaging, Asgar Ali Hospital, Dhaka, Bangladesh \\ ${ }^{2}$ Deptt. of Radiology and Imaging, Sir Salimullah Medical College and Mitford Hospital, Dhaka, Bangladesh \\ ${ }^{3}$ Department of Radiology and Imaging, Impulse Hospital, Dhaka, Bangladesh \\ e-mail:iffatu@gmail.com
}

\begin{abstract}
Diagnostic usefulness of multidetector computed tomography (MDCT) scan in the evaluation of neck mass was assessed in this study in terms of sensitivity, specificity, positive and negative predictive value and accuracy in respect to characterise the lesion as benign and malignant, based on different parameters by comparing computed tomography and histopathological findings. This cross sectional study was carried out on 57 patients in the Department of Radiology and Imaging, Sir Salimullah Medical College and Mitford Hospital, Dhaka from January 2014 to December 2015, with a 16 slice multidetector CT scanner and the reports were compared to histopathological diagnosis. Out of 57 patients, $37(64.9 \%)$ and $20(35.1 \%)$ were diagnosed as malignant and benign lesions respectively, with a sensitivity of $94.6 \%$, specificity $95 \%$, positive predictive value $97.2 \%$, negative predictive value $90.5 \%$ and diagnostic accuracy of $94.7 \%$. As an imaging modality, thus, MDCT proved to be a useful tool for assessing and characterisation of neck mass as benign and malignant, especially in this part of the continent, and is essential in planning surgical approach and predicting prognosis.
\end{abstract}

\section{Introduction}

Cancer of the head and neck is the sixth most frequent cancer worldwide that presents in patients of all ages. ${ }^{1}$ Underlying causes may be congenital, inflammatory, benign and malignant neoplasm. In children, $90 \%$ of neck masses are benign and more than $95 \%$ are due to infection. In adult older than 40 years of age, a malignant tumor may be expected in more than $50 \%$ cases. In adult, $80 \%$ of nonthyroid, nonsalivary glands are neoplastic, and $80 \%$ neoplastic masses are malignant. ${ }^{2}$ Among the imaging modalities computed tomography (CT) with its unique capacity to display osseous and soft tissue details has become an indispensable tool in the evaluation of patient with a neck mass. ${ }^{3}$ Multidetector computed tomography permitted the rapid scanning of large volumes of tissue during quiet respiration and it is less susceptible to patient motion and resulted in improved resolution, it is also readily available. Other advantages of this technique include improved temporal resolution into arterial and venous phases, volume acquisition of data enabling convenient retrospective reconstructions, and unlimited reformations leading to increased lesion conspicuity. Spiral CT is standard one for imaging neck tumors. Secondary coronal reconstructions of axial scans are very helpful in the evaluation of crossing the midline by small tumors of the palate or tongue base. However, CT requires iodinated contrast and ionising radiations. ${ }^{3}$ Currently, fine needle aspiration biopsy is the standard of diagnosis for neck masses. FNAB separates inflammatory and reactive processes that usually do not require surgery from neoplastic lesions, either benign or malignant. The main purpose of the head and neck imaging is to evaluate the true extent of disease to determine the best surgical and therapeutic needs. ${ }^{3}$ The overall accuracy of clinical examination of the neck was $70 \%$ versus $93 \%$ by $\mathrm{CT}$, stated in a study by Stevens et al. ${ }^{4}$ In a study, Atula et al demonstrates among 86 patients with clinically no palpable mass in the neck, CT showed lymph nodes fulfilling the radiologic criteria for malignancy in $27 \%$, MRI in $17 \%$ and US in $14 \%$ cases. ${ }^{5}$ In another study, Shetty et al showed that the specificity of CT to diagnose metastatic neck node is $93.7 \% .{ }^{6}$ Gupta et al studied 45 patients of neck mass lesions with multislice multidetector CT and found the accuracy of MDCT is very high in detecting benign and malignant lesions, $97 \%$ and $100 \%$ respectively. ${ }^{7}$ However, the current study was designed to assess the diagnostic usefulness of multi-detector computed tomography scan in the evaluation of neck mass in terms of sensitivity, specificity, positive and negative predictive value 
and accuracy in respect to characterize the lesion as benign and malignant, based on different parameters by comparing computed tomography and histopathological findings.

\section{Materials and Methods}

This cross sectional study was carried out on 57 patients with neck mass in the department of Radiology and Imaging Sir Salimullah Medical College and Mitford Hospital, Dhaka, from January 2014 to December 2015, with a 16 slice multi detector CT scanner and the reports were compared to histopathological diagnosis allocating the lesions as benign or malignant and performance of diagnostic test was calculated.

MDCT criteria of benign and malignant lesions: Referring to the study, masses reported as malignant lesions on MDCT showed an ill defined margin, density equal to or more than that of muscle, presence of necrosis, blurring of fat plane, heterogeneous enhancement, lymphadenopathy with malignant criteria like enlarged size $(>1 \mathrm{~cm})$, rounded contour, absence of hilum, eccentric cortical thickening, necrosis, ill-defined margins with infiltration of surrounding structures and loss of fat planes, infiltration into surrounding tissue and bones or cartilage erosion. ${ }^{1}$ Benign lesions were characterized by the presence of a well defined margin, density less than that of muscle, coarse globular or rim like calcification, displaced but maintained fat plane and showing homogenous contrast on post-contrast studies. In inflammatory lesions (benign) ill defined margin, a density less than that of muscle, presence of necrosis, perilesional blurred fat plane, heterogeneous or peripheral thick rim enhancement and lymphadenopathy with benign criteria enlarged size $(>1 \mathrm{~cm})$ but oval contour, presence of hilum, well defined margins with no infiltration of surrounding structures, no loss of fat planes and usually no necrosis. ${ }^{1}$

Analysis of data:All the relevant collected data were compiled on a master sheet first. Then percentages were calculated to find out proportion of the findings. $\mathrm{Z}$ test of proportion was done to see the significance of differences. A $p$ value of $<0.05$ was considered as significant. Further statistical analysis of the results was done using SPSS (statistical package for social sciences) For the validity of study outcome, sensitivity, specificity, positive predictive value, negative predictive value and accuracy of MDCT scan in the diagnosis of neck mass were calculated after confirmation of diagnosis by histopathology.

\section{Results}

Out of the total 57 patients with clinical sign symptoms of neck mass $37(64.9 \%)$ were diagnosed as malignant and the remaining 20 $(35.1 \%)$ as benign. Mean age of the patients was $46.08 \pm 20.45$ years ranging from $02-82$ years with male to female ratio of $1.9: 1$. The largest percentage $(26.3 \%)$ was in 51-60 year, followed by $41-50$ year $(17.5 \%)$ and then $61-70$ year Fourteen percent patient was in paediatric age group (less than 12 years old). Among all malignant lesions, 58\% cases were noted in 41-60 years of age group, while the benign lesions were distributed evenly among all age groups. Out of 57 patients with neck mass symptomatology most common clinical presentation was neck swelling in both benign (95\%) and malignant lesion (89\%) followed by dysphagia. Distribution of the lesions were noted according to neck spaces, among all the neck spaces visceral spacewas the most common space to be involved in both benign (25\%) and malignant lesion (46\%). Of the lesions involving the suprahyoid neck spaces the maximum number of lesions were recorded in the pharyngeal mucosal space $(n=10)$ followed by those in the parapharyngeal space $(n=7)$. In the infrahyoid neck, the predominant lesions $(n=22)$ were observed in visceral space followed by posterior cervical space $(\mathrm{n}=8)$ and then anterior cervical space $(n=5)$. It is mentionable that many of the lesions occupied more than single neck space (table I).

Table I: Distribution of patients by percentage of benign and malignant neck masses according to neck spaces

\begin{tabular}{lccc}
\hline Space & Patient (\%) & Benign (\%) & Malignant (\%) \\
\hline PCS & $8(14)$ & $2(10)$ & $6(16)$ \\
ACS & $5(9)$ & $5(25)$ & $0(0)$ \\
VS & $22(39)$ & $5(25)$ & $17(46)$ \\
PMS & $10(17.5)$ & $1(5)$ & $9(24)$ \\
PPS & $7(12.3)$ & $4(20)$ & $3(8)$ \\
RPS & $1(1.75)$ & $1(5)$ & - \\
SMS & $2(3.5)$ & $1(5)$ & $1(2.7)$ \\
MS & $1(1.75)$ & - & 1 \\
PEVS & $3(5.3)$ & $3(15)$ & - \\
CS & $3(5.3)$ & $3(15)$ & - \\
Oral cavity & $2(3.5)$ & - & $2(5.5)$ \\
PS & $5(9)$ & $2(10)$ & $3(8)$ \\
\hline
\end{tabular}

PCS: Posterior cervical space, ACS: Anterior cervical space, VS: Visceral space, PMS:Pharyngeal mucosal space, PPS: Parapharyngeal space, RPS: Retropharyngeal space, SMS: Submandibular space, MS: Masticator space, PEVS: Perivertibral space, CS: Carotid space, Oral cavity, PS: Parotid space. 
Margin was found mostly (94\%) ill defined in malignant lesions and well defined (80\%) in benign lesions. Density was iso (70\%) and hyper $(30 \%)$ than that of adjacent muscles in malignant lesions but mostly hypo (65\%) to iso (35\%) in benign lesions. Malignant lesions were mostly solid in nature $(87 \%)$ with only $13 \%$ were mixed solid and cystic whereas benign lesions were solid $(45 \%)$, cystic $(35 \%)$ and mixed $(20 \%)$. Malignant lesions were mostly heterogeneously enhancing $(81.1 \%)$. Most of the malignant lesions show fat plane obliteration or blurring $(86.5 \%)$ whereas benign lesions had displaced but maintained surrounding fat plane $(80 \%)$. Most of the criteria of malignant and benign lesions were statistically significant $(p<0.05)$. In this study, MDCT in comparison with histopathology showed that it has the ability to evaluate the malignant and benign tumors of the neck with a sensitivity $94.6 \%$, specificity $95 \%$, positive predictive value $97.2 \%$, negative predictive value $90.5 \%$ and diagnostic accuracy of $94.7 \%$ (table II).

Table II: MDCT and histopathological comparison of benign versus malignant lesions

\begin{tabular}{ccccc}
\hline \multicolumn{4}{c}{ Histopathological Diagnosis } \\
\hline \multirow{4}{*}{ CT diagnosis } & Malignant & Benignt Tota \\
& Malignant & 35 & 1 & 36 \\
& Benign & 2 & 19 & 21 \\
& Total & 37 & 20 & 57 \\
\hline
\end{tabular}

Sensitivity $=94.6 \%, \quad$ Specificity $=95 \%, \quad$ Positive predictive value $=97.2 \%$, Negative predictive value $=90.5 \%$, Accuracy $94.7 \%$.

\section{Discussion}

Shrestha et al in his study found that $29 \%$ of the patient of neck mass was in the age group 51-60 years followed by 41-50 years and then 61-70 year. ${ }^{8}$ Among all $29 \%$ of malignant lesions were in 41-60 years age group. Charan et al found that the largest age group of patients with neck mass was in the age group 46-60 years (29\%) followed by $31-45$ years $(24 \%)$ with higher incidence of malignant lesions between 46-60 years and also found the male female ratio is $2.1: 1 .^{3}$ So, the present study was consistent with other studies in respect to age and sex distribution as well as distribution of malignant lesions among all age group.
Most common presenting complaints were consistent with Charan et al who found neck swelling as commonest presentation in both benign (92\%) and malignant (93\%) lesions. ${ }^{3}$ Of the lesions involving the suprahyoid neck spaces, the maximum number of lesions were encountered in pharyngeal mucosal space $(\mathrm{n}=10)$ of which $70 \%$ $(n=7)$ was squamous cell carcinoma in this study, consistent with the findings of Neel and Slavitt who reported squamous cell carcinoma to constitute adult nasopharyngeal malignancies. Shrestha et al also found squamous cell carcinoma constituting $80 \%$ of pharyngeal mucosal space lesion. ${ }^{8}$

The most common lesion occurring in the parapharyngeal space in this study $29 \%$ were SCC while $29 \%$ were benign tumor like schwannoma and basal cell adenoma of the minor salivary gland, $14 \%$ was mucoepidermoid carcinoma extended from parotid space, $14 \%$ reactive lymphadenitis and $14 \%$ were abscess. Hughes et al in his study of 172 patients with parapharyngeal neoplasm found pleomorphic adenoma $(40 \%)$ as the commonest lesion. ${ }^{10}$ While Bozza et al reported $66.6 \%$ as benign (pleomorphic adenoma) and $33.3 \%$ as malignant squamous cell carcinoma (SCC) metastasis from occult primary and extending from tonsil region and adenocarcinoma of parotid. ${ }^{11}$ The disparity between present and above studies could be due to the fact that malignancy, infection and abscess are common in Bangladesh due to the life style pattern, poor oral hygiene and lack of awareness. Also the patients present late until they face severe problem with the lesion. Small sample size could partly be responsible for discrepancies between our studies. One study in Nepal reflected the above problems in this subcontinent \& reported SCC andabscess accounting for $29.1 \%$ each, which is consistent with the present study. ${ }^{8}$ One case of schwannoma was reported in present study, which corresponds with Som et al who reported that schwannomas accounted for $80 \%$ of neurogenic tumors. ${ }^{12}$

Boucher et al has reported abscesses in $96 \%$ of the cases involving the retropharyngeal space, which is consistent with present study. ${ }^{13}$ Peel and Gnepp reported $70-80 \%$ tumors involving the parotid gland to be pleomorphic adenoma, $30 \%$ mucoepidermoid and $28 \%$ adenocarcinoma, which largely corresponds with the present study. ${ }^{14}$ Among the lesions involving the submandibular 
space $(n=2)$, abscess $(50 \%)$ was predominant lesion, which correlates most of the literatures.

Vander et al reported the incidence of colloid goiter to be $3-5 \%$ in the general population corresponding to present study. ${ }^{15}$ Hedinger et al found in his study papillary carcinoma accounting as $80-90 \%$ of thyroid carcinoma, which also close to the present study finding. ${ }^{16}$ The partial disparity between the present and this study can be attributed partly to the fact that the sample size of the present study was small and in endemic thyroid area the percentage of papillary carcinoma is significantly less than that of nonendemic area, reported by Hedinger et al. ${ }^{16}$ Female preponderance was also seen in thyroid lesions with a ratio of female male is $1.8: 1$ which corresponds to most of the literature.

The malignant tumors involving larynx andhypopharynx were all squamous cell carcinoma, which corresponds to the study done by Becker, who reported over $90 \%$ of lesions in this space to be SCC. Lymph nodes found to be the most common site to be involved in present study. Charan et al found in his study that in $47 \%$ patients with neck nodes the primary was known, while in nodal metastasis from unknown primary was identified in $13.7 \%$ of the malignant cases and lymph node was suspected as a primary site of malignancy in $8.3 \%$, which was consistent with the present study. ${ }^{3}$ In the present study, the main differentiating features between benign and malignant lesions were margin, density of lesions, nature, calcification, necrosis, enhancement pattern, fat plane, involvement of vessels, bone/cartilage erosion and lymphadenopathy, were consistent with those described by David and Kathleen. ${ }^{18}$ Percentage of different CT criteria of this study like margin of the lesions, fat plane obliteration, necrosis, density, enhancement, presence of calcifications were consistent with the study of Shrestha et al and Charan et al., ${ }^{3,8}$ Out of all malignant cases $44 \%$ were squamous cell carcinoma. $12 \%$ of the lesions among the total 57 patients were of an infective origin and $23 \%$ of all the lesions were benign and congenital cystic lesions. The findings were consistent with the findings of Shrestha et al. ${ }^{18}$

The present study showed that, MDCT is significantly precise in distinguishing malignant from benign lesions of the neck. But radiological features of the most of the malignant tumors are nonspecific and their differentiation from each other and thus reaching a definitive diagnosis is difficult. However, on MDCT scan image a particular malignant tumor can be assigned to a particular neck space, thus reaching a narrow differential diagnosis pertaining to that space and the age of patient. By defining the extent of lesions and involvement of adjacent structures, MDCT helps in the planning of treatment and the portal for radiotherapy as well.

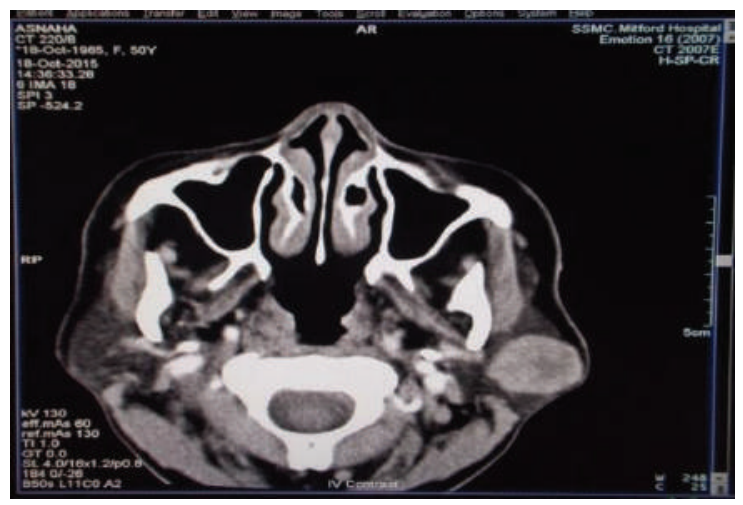

Figure 1: Postcontrast axial CT neck- pleomorphic adenoma of left parotid gland.

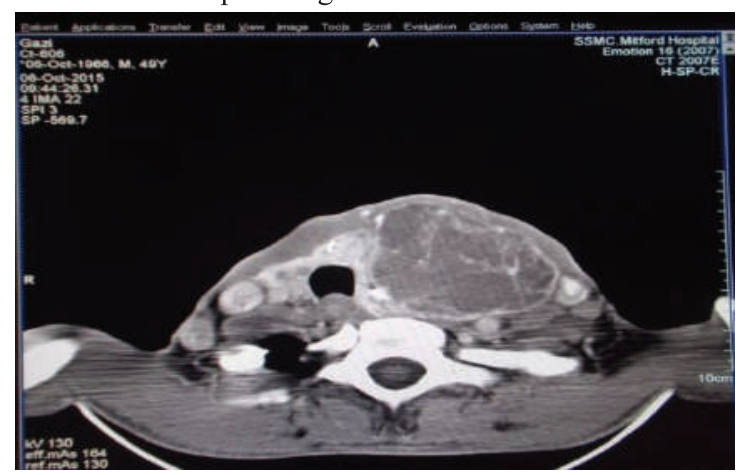

Figure 2: Postcontrast axial CT neck-papillary carcinoma of thyroid with metastatic carotid space lymphadenopathy.

\section{Conclusion}

As an imaging modality, multi detector computed tomography scan has the ability to evaluate the malignant and benign tumors of the neck with a sensitivity $94.6 \%$, specificity $95 \%$, positive predictive value $97.2 \%$, negative predictive value $90.5 \%$ and diagnostic accuracy of $94.7 \%$. The other advantages of MDCT are its availability, relatively low cost, short examination time thus important for paediatric and non cooperative patients that is reflected in current study as no major information loss was noted in any case due to patient motion. MDCT also provides excellent bony detail and the anatomic landmarks at the skull base and tumoral calcification and it is also a 
noninvasive test. Hence, it may be concluded that the accuracy of MDCT in the evaluation of neck mass is reliable in comparison to histopathology considering the later as standard but an invasive procedure. Current study included all neck mass lesions irrespective of their diagnosis and histopathological variety. It would have better if individual type of lesion could be considered separately. Because different pathological conditions respond to different diagnostic procedures in different manner. In most of the studies sensitivity and specificity for individual type of lesion were sought. But it is extremely difficult to enroll sufficient subjects of such pathology for a study. A largescale study with great logistic support and adequate randomisationis thus recommended.

\section{References}

1. Vogl TJ. Cervical adenopathy and neck masses.In : John. R. Hagga, et al. ed. CT and MRI of the whole body. Vol.1. $5^{\text {th }}$ ed. Philadelphia: Mosby Elsevier, 1993: 639-67.

2. ReibalJF. The Patient with a Neck Mass. Comprehensive Therapy. 1997; 23: 737-41.

3. Charan I, Kapoor A, Kumar N, Jagawat N, Singhal, M.K., Kumar, H.S. Evaluation of neck Mass with Computed Tomography: An Observational Study. Int J Sci Stud. 2014; 2: 118-122.

4. Stevens, MH, Harnsberger HR, Mancuso AA, Davis RK, Jhonson LP, Perkin JL. Computed Tomography of cervical lymph nodes. Staging and management of head and neck cancer. 1985; 11: 735-39.

5. Atula TS, Varpula MJ, Kurti TJ, Klemi PJ,Grenman, R. Assessment of cervical lymph node status in head and neck cancer patients: palpation, computed tomography and low field magnetic resonance imaging compared with ultrasound-guided fine-needle aspiration cytology. Eur J Radiol. 1997; 25: 152-61.

6. Shetty D, Jayade BV, Joshi SK, Gopalkrishnan K. Accuracy of palpation, ultrasonography, and computed tomography in the evaluation of metastatic cervical lymph nodes in head and neck cancer. Indian Journal of Dentistry. 2015;6:121-24.

7. Gupta P, Satish K, Gopesh M, Vineeta R. Role of multislice spiral CT in the evaluation of neck masses. JIMSA. 2013; 26: 51-54.

8. Shrestha MK, Ghartimagar D, Ghosh A. CT evaluation of neck mass. J Nepal Med Assoc,. 2011; 51: 164-70.

9. Neel HB, Slavitt DH. Nasopharyngeal cancer. In: Baily BI, editor. Head and neck surgery: Otolaryngology. Philadelphia: JB Lippincott,.1993; 1257-60.

10. Hughes KV, Olsen KD, Mccaffrey TV. Parapharyngeal space neoplasms. Head Neck. 1995; 17: 124-30.

11. Bozza F, Vigil MG, Ruscito P, Marzetti A, Marzetti F. Surgical management of parapharyngeal space tumor: results of 10 year follow-up. Acta Otorhinolaryngol Ital.2009; 29: 10-15.

12. Som PM, Sacher M, Stollman AL, Biller HF, Lawson W. Common tumors of the parapharyngeal space: refined imaging diagnosis.Radiology.1988;169:81-85.

13. Boucher C, Dorion D, Fisch C. Retropharyngeal abscesses. A clinical and radiological correlation. J Otolaryngeal. 1999; 28: 134-37.

14. Peel RZ, Gnepp DR. Disease of the salivary glands In: Barnes L, editor. Surgical pathology of head and neck vol.1. New York: Marcel Dekker, 1985; 535-52.

15. Vander JB, Gaston EA, Dawber TR. The significance nontoxic thyroid nodules. Ann Intern Med. 1968; 69: 537-40.

16. Hedinger C. Geographic pathology of thyroid diseases. Pathology-Research and Practice. 1981; 171: 285-92.

17. Becker, M. Larynx and Hypopharynx. Radiologic clinics of North America. 1998; 36: 891-19.

18. David MY, Kathleen M. Head and neck lesions. Radiologic-pathologic correlations. Radiologic clinics of North America. 1998; 36: 983-14. 